\title{
Growth, Structural and Thermoelectric Properties of Tungsten Diselenide Crystals
}

\author{
P. R. Patel1, V. M. Pathak2 \\ ${ }^{1}$ Smt S. M. Panchal Science College, Talod - 383 215, Gujarat, India \\ ${ }^{2}$ Department of Physics, Sardar Patel university, Vallabh Vidyanagar - 388 120, Gujarat, India
}

Abstract: Crystals of Tungsten diselenide (WSe $e_{2}$ ) have been grown by direct vapour transport (DVT) technique using micro processor controlled dual zone horizontal furnace. The chemical composition and structure of grown crystals were confirmed using energy dispersive analysis of X-ray (EDAX) and X-ray diffraction (XRD). In the present investigation thermoelectric power measurements (TEP) have been carried out on the grown crystals. Different electrical transport parameters of semiconductors have been determined and discussed in the paper.

Keywords: WSe $e_{2}$, Structural, Electrical, EDAX, XRD.

PACS: $71.18+y, 71.20 .-b, 71.20 \mathrm{Nr}, \mathrm{,} \mathrm{73.90+F}$

\section{Introduction}

In recent years, group- $V I$ semiconductors have attracted much interest in their applications particularly in the area of solar cells. WSe $e_{2}$ is a member of the compounds of VI-A and VI-B group. It has stacked layer structure of M-X-M type which is an extra incentive for device research. Here $M$ is transition metal e.g. tungsten and $\mathrm{X}$ is chalcogen e.g. Selenium [1,2]. Amongst layered TMDCs, DVT grown $\mathrm{WSe}_{2}$ crystals have been less studied for their thermoelectric properties in a wide temperature range.

In the present investigation thermoelectric properties of the well characterized grown crystals of $\mathrm{WSe}_{2}$ have been done. Electrical transport parameters like carrier concentration, Fermi energy, density of states and effective mass of carriers have also been determined and discussed in the paper.

\section{Experimental}

Crystals of Tungsten diselenide ( $\mathrm{WSe}_{2}$ ) have been grown by direct vapour transport (DVT) technique using dual zone horizontal furnace [3]. The chemical composition was identified using energy dispersive analysis of X-ray (EDAX). XRD analysis was carried out by X-ray Diffractometer for the determination of structure. The thermoelectric power measurements (TEP) have been carried out on the grown crystals with the help of TEP measurement setup developed in our laboratory.
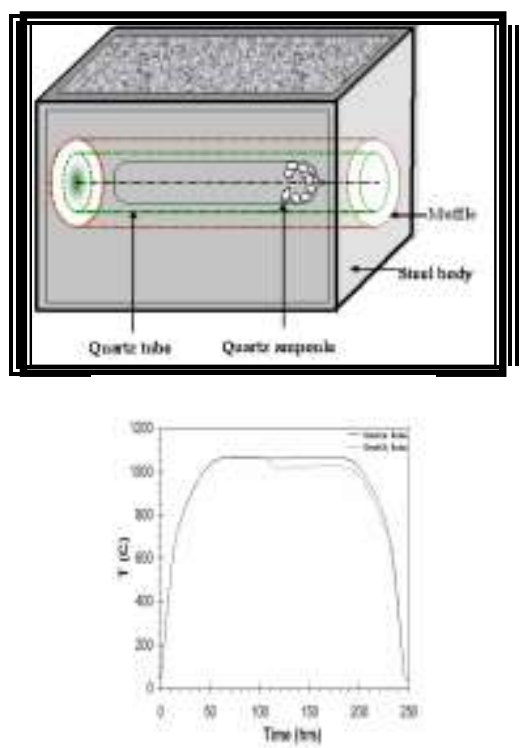

Fig.1. The temperature profile used for the growth of $\mathrm{WSe}_{2}$.

The thermoelectric power measurements (TEP) have been carried out on the grown crystals with the help of TEP measurement setup developed in our laboratory. The sample with area $3 \times 3 \mathrm{~mm}^{2}$ was used.. The 
measurements were taken in the temperature range of $333 \mathrm{~K}$ to $473 \mathrm{~K}$ and the temperature difference $(\Delta \mathrm{T})$ between the ends of the sample was kept to be $10 \mathrm{~K}$.

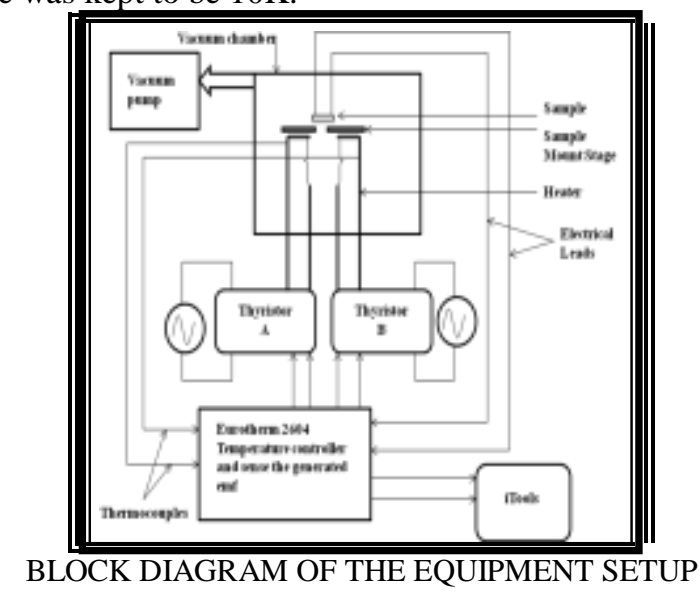

III. Results And Discussions

The stoichiometry of the grown crystals was analyzed by EDAX and it is found that the grown crystals posses nearly perfect stoichiometry with chemical formula $\mathrm{WSe}_{2}$.

Fig.1 shows the X-ray diffractogram of $\mathrm{WSe}_{2}$. crystal. XRD analysis shows that crystals posses hexagonal structure. For this hexagonal structure lattice parameters $\mathrm{a}, \mathrm{b}$ and $\mathrm{c}$ were determined. The obtained values are $\mathrm{a}=\mathrm{b}=3.28 \AA$, and $\mathrm{c}=13.00 \AA$. These observed lattice parameters of as grown crystals are in good agreement with reported JCPDS data [4].

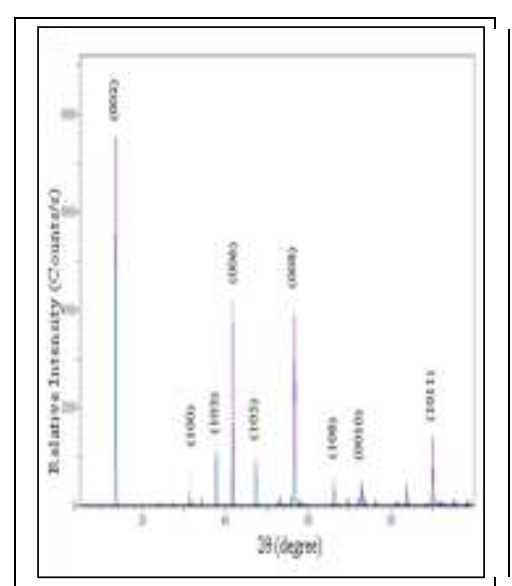

FIGURE 1. X-ray diffractogram of $\mathrm{WSe}_{2}$ crystal.

The results of TEP measurements as shown by variation of thermoelectric power (S) with temperature in the range $333 \mathrm{~K}$ to $473 \mathrm{~K}$ have been shown in fig 2 (a).The positive values of ' $\mathrm{S}$ ' shows that the crystals possess p-type conductivity. To analyze the temperature dependence of thermoelectric power of a p-type semiconductor, the expression given by Mohanchandra and Uchil [5] and H. J. Goldsmid [6] has been used. It is given as

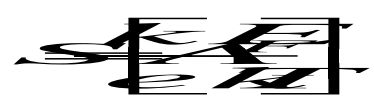

where $\mathrm{k}$ is Boltzmann constant, e is electronic charge, $\mathrm{A}=(5 / 2-\mathrm{C})$ is the scattering coefficient which varies from 0 to 4 depending on the scattering process,$C$ is the scattering parameter and $E_{F}$ is the separation of Fermi level from the top of the valance band.

As the carrier concentration depends on $\mathrm{E}_{\mathrm{F}}$ in the crystal and for a small temperature range, $E_{F}$ can be considered as fairly constant, equation (1) can be expressed as

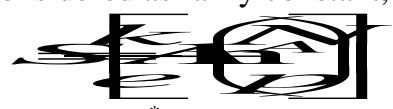

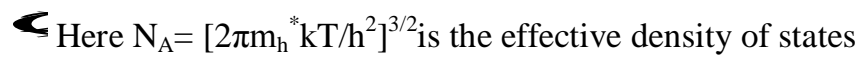
in which $\mathrm{m}_{\mathrm{h}}{ }^{*}$ is the effective mass of holes and $\mathrm{h}$ is the Plank's constant.

Considering constant $\mathrm{E}_{\mathrm{F}}$, the plot of thermoelectric power (S) against the reciprocal of temperature gives a straight line as shown in fig.2 (b). 


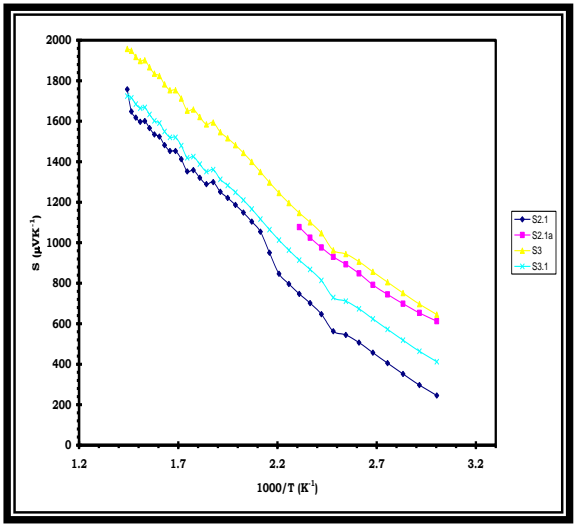

FIGURE 2 (a). Variation of thermoelectric power vs temperature and (b). Variation of thermoelectric power as a function of inverse of temperature

From the slope the value of $\mathrm{E}_{\mathrm{F}}$ is found to be $0.0368 \mathrm{eV}$. Using the value of carrier concentration $\left(6.67 \times 10^{18} \mathrm{~cm}^{-3}\right)$ obtained from Hall Effect measurement, effective density of states $\mathrm{N}_{\mathrm{A}}$ was also calculated and is found to be $6.679 \times 10^{24} \mathrm{~m}^{-3}$. Using this value the effective mass was calculated and is found to be $7.597 \times 10^{-31}$ $\mathrm{kg}$. These calculated values for DVT grown $\mathrm{WSe}_{2}$ crystal are close to the values reported in the literature [7].

\section{Conclusions}

The EDAX analysis shows that the grown crystals are nearly perfect in stoichiometry. The X-ray analysis shows that crystals posses hexagonal structure. The values of the computed lattice parameters for the grown crystals are $a=b=3.28 \AA$ and $c=13.00 \AA$. The positive values of thermoelectric power show that the crystals possess p-type conductivity. The values of Fermi energy $E_{F}$, Density of states $N_{V}$, effective mass $\mathrm{m}_{h} *$ obtained for crystal are close to the values reported in the literature.

\begin{tabular}{||cccc||}
\hline Sample & $\mathbf{E}_{\mathbf{F}}(\mathbf{e V})$ & $\mathbf{A}$ & $\mathbf{S}$ \\
\hline WSe $_{2}$ & 0.019 & 4.20 & -1.71 \\
\hline
\end{tabular}

Table 1. $\mathrm{E}_{\mathrm{F}}$ and values of scattering parameter $s$ for $\mathrm{W}_{-} \mathrm{Se}_{2}$ crystals

\section{References}

[1] M. P. Deshpande, M. K. Agarwal, P. D. Patel and D. Laxminarayana, Journal of materials science letters, 18, 233 (1999).

[2] Armin Retterberger and Paul Leiderer, Phys. Rev. B 56, 12092 (1997)

[3] M. K. Agarwal ,P.D.Patel, and S. K. Gupta, Journal of Crystal Growth 129,559 (1993).

[4] JCPDS,International centre for diffraction data, PDF-2 File No.38-1388 (1997).

[5] K. P. Mohanchandra, J. Uchil J, Solid Films, 305, 124 (1997)

[6] H. J. Goldsmid, Apllications in Thermoelectricity, Methuen Monograph, London (1950).

[7] G. K. Solanki, D. Gujarathi, M. P. Deshpande, D. Laxminarayana and M. K. Agaawal Cryst.Res. Techol. 43(2), 179 (2008). 